\title{
Laryngomalacia and inspiratory obstruction in later childhood
}

\author{
G J SMITH AND D M COOPER \\ Department of Pulmonary Medicine, Adelaide Children's Hospital, South Australia
}

SUMmary Pulmonary function and symptoms were reviewed in 20 children in whom laryngomalacia had been diagnosed by direct laryngoscopy in infancy. Most children developed stridor in the first 2 weeks, but 3 children did not develop it until age 3 months. The mean duration of stridor was 4 years 2 months, with a range of 4 months to 12 years 7 months. Six children had marked posseting as infants. Airway dynamics were assessed by flow volume loops. All except one had normal expiratory flow volume curves. Inspiratory abnormalities were detected in 7 children; 6 were assessed as having variable extrathoracic inspiratory obstructions and 1 had a fixed obstruction consistent with subglottic stenosis. The mean ratios of maximal inspiratory flow at $50 \%$ of vital capacity divided by forced vital capacity in the laryngomalacia and control groups differed significantly, as did the mean ratios of maximal expiratory flow to maximal inspiratory flow at $50 \%$ of forced vital capacity. Laryngomalacia is not necessarily a benign disorder of limited duration; there may be persisting inspiratory obstruction in later childhood.

Laryngomalacia is characterised by inspiratory stridor beginning at birth or soon after it, which is exacerbated by crying and respiratory infections, and at times is complicated by feeding problems. It has a benign prognosis with spontaneous resolution of symptoms towards the end of the second year of life.

Phelan et al. ${ }^{1}$ reviewed 51 children with laryngomalacia and performed body plethysmography in 10 , demonstrating an increase in both inspiratory and expiratory resistance with an inspiratory/ expiratory resistance ratio of $4 \cdot 1$ compared with a normal value of $0 \cdot 7$.

The flow volume loop has been used to assess airways obstruction in adults ${ }^{2}$ and patterns of fixed and variable extrathoracic and intrathoracic obstruction have been described. ${ }^{3}$ The ratio of forced expiratory flow to forced inspiratory flow at $50 \%$ of vital capacity ( $\dot{\mathrm{V}} \max 50$ (E)/ $\dot{\mathrm{V}} \max 50$ (I) or mid VC ratio), has been shown to be particularly useful in assessing extrathoracic obstruction. ${ }^{3-6}$

\section{Patients and methods}

A 9-year-old boy (Case 1) presented because of pronounced effort-associated dyspnoea which had been present for at least 12 months. He was the product of a normal pregnancy. At 4 days of age stridor had been noted, and at 1 week direct laryngoscopy demonstrated laryngomalacia. The stridor persisted for 12 months, and there were no further respiratory symptoms until age 8 , when he underwent a considerable increase in linear growth and assumed a more active life style. On examination he was greater than the 97th centile for height and weight, normally proportioned, and free of chest deformity or other physical abnormality at rest. Treadmill exercise produced intense dyspnoea from which he recovered in less than 5 minutes. Pulmonary function test results showed fixed central airways obstruction consistent with subglottic stenosis. A barium swallow was normal, and laryngeal tomography showed a constricted laryngeal additus and rima glottidis.

The records of the Adelaide Children's Hospital were reviewed for cases of endoscopically-confirmed laryngomalacia between 1960 and 1973. There were 96 such cases. Twenty-seven children were excluded from the study because they could not be expected to

Abbreviations:

FVC forced vital capacity

VC vital capacity

PEFR peak expiratory flow rate

$\mathrm{FEV}_{1}$ forced expiratory volume in one second 
perform lung function tests satisfactorily ( 25 were under 5 years, and 2 were mentally retarded), 24 lived some distance from Adelaide and it was not thought reasonable to recall them for assessment, and one child had died from drowning. Letters were sent to the 45 remaining children, and the 20 who replied comprised the study group. The mean age at the time of study was 8 years 1 month (range 5 years 4 months to 12 years 7 months). A control group of 29 healthy children was studied for comparison.

Parents of the children completed questionnaires concerning clinical features and the natural history of the disorder. Questions were asked about the age of onset and duration of stridor, exacerbating factors, whether cyanosis or dyspnoea was ever present, and the frequency of respiratory infections. Obstetric, neonatal, and family histories were obtained. Developmental milestones were documented, as was information concerning feeding problems in the first year of life.

Pulmonary function was assessed using an Ohio 842 spirometer in 19 children in the study group. One child was found to be slightly mentally retarded and was unable to co-operate. A series of maximal expiratory and inspiratory efforts was performed to obtain complete flow volume loops (Fig. 1). The shape of the loop was noted and forced vital capacity (FCV), expiratory flow at $50 \%$ and $25 \%$ of FVC ( $\dot{\mathrm{V} m a x} 50$ (E) and $\dot{\mathrm{V} m a x} 25$ (E) ), inspiratory flow at $50 \%$ of FVC (V $\max 50$ (I)), peak expiratory flow rate (PEFR), and forced expiratory volume in one second $\left(F E V_{1}\right)$ obtained. All flows were standardised by dividing them by observed FVC. V $\max 50$ (E) was divided by $\dot{V} \max 50$ (I), to obtain the mid VC ratio. FVC and $\mathrm{FEV}_{1}$ were expressed as a percentage of normal values using data of Polgar and Promadhattavedi. ${ }^{7}$

\section{Results}

Questionnaires. In most instances stridor had appeared by age 2 weeks (Fig. 2). However, in 5 children it appeared later. Fig. 3 shows the duration of stridor. In $9(47 \%)$ children stridor had persisted beyond 2 years of age, the eldest of these being a girl of 12 years 7 months. Stridor was exacerbated by respiratory infections in 14, and by feeding in 9 . Thirteen suffered frequent upper respiratory tract infections and 12 had frequent lower respiratory infections as judged by the parents' recollection. One child had recurrent aspiration pneumonia. Obstetric and family histories were normal in all cases. Six children had persistent posseting consistent with gastro-oesophageal reflux, noted in case records but not radiologically confirmed, and which

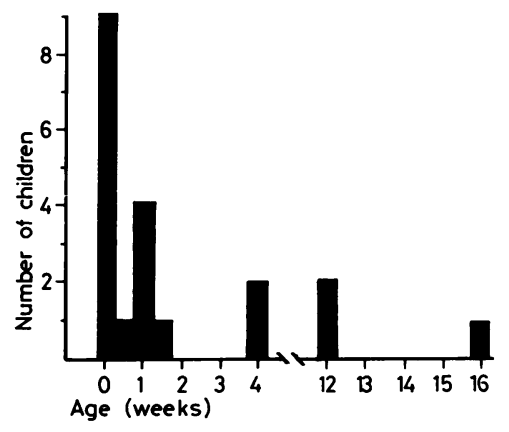

Fig. 2 Age of onset of stridor.

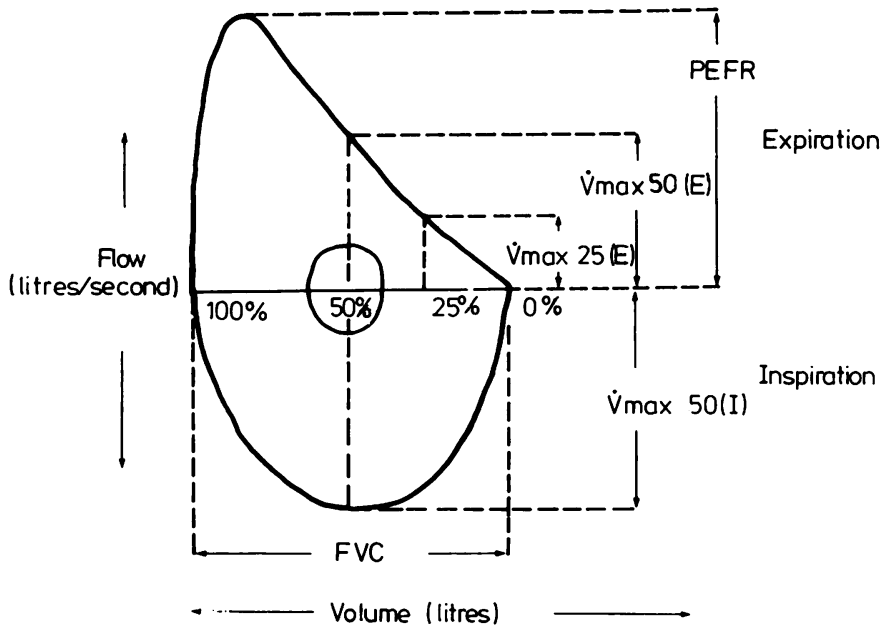

Fig. 1 Normal flow volume loop showing indices assessed. 


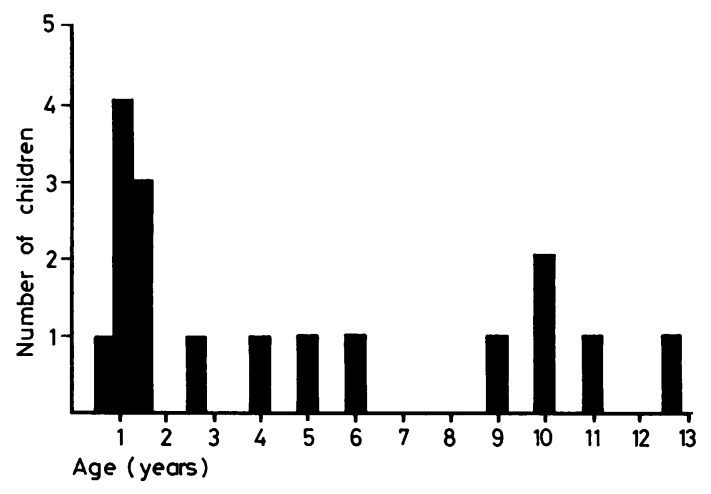

Fig. 3 Age at which stridor went.

had resolved in all cases by 12 months. Developmental milestones were normal in all but one child who was slightly mentally retarded and had epilepsy following aspiration pneumonia and hypoxia at birth.

Pulmonary function tests. Seven children had abnormally shaped flow volume loops. Fig. 4 shows the loop from Case 1. The shallow flattened inspiratory curve and attenuated expiratory curve are consistent with subglottic stenosis which was confirmed by laryngeal tomography. The PEFR was $66 \%$ of predicted normal and mid VC ratio was $1 \cdot 6$. A further 6 children had loops showing shallow inspiratory curves but normal expiratory curves (Fig. 5), producing a high mid VC ratio. The Table shows mean values and standard deviations for the indices assessed in the patients with laryngomalacia and in

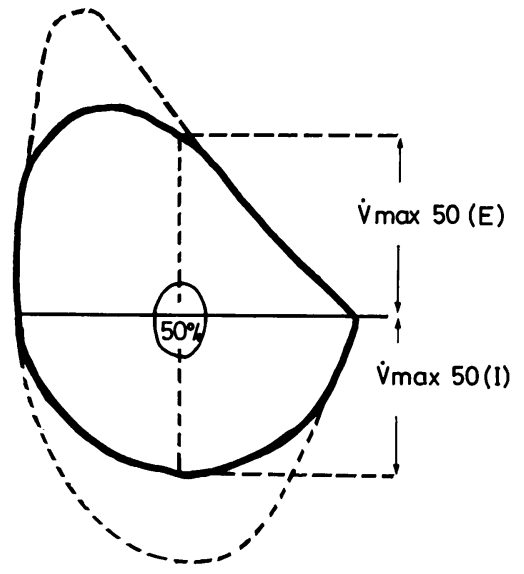

Fig. 4 (Case 1.) Flow volume loop showing an attenuated expiratory curve, shallow inspiratory curve, reduced PEFR, and high mid VC ratio (1.6). Dotted lines show appropriate normal curves.

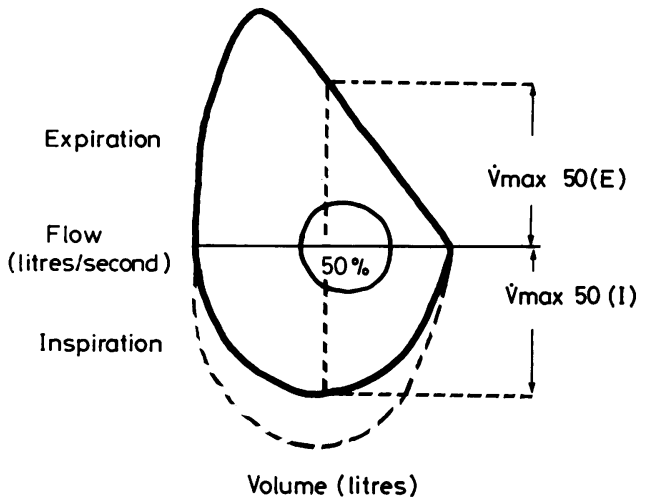

Fig. 5 Representative abnormal flow volume loop in a patient with laryngomalacia. Dotted line shows an appropriate normal inspiratory curve.

Table Pulmonary function tests

\begin{tabular}{|c|c|c|c|c|}
\hline & \multicolumn{2}{|c|}{$\begin{array}{l}\text { Laryngomalacia } \\
(n=19)\end{array}$} & \multicolumn{2}{|c|}{$\begin{array}{l}\text { Controls } \\
(n=29)\end{array}$} \\
\hline & Mean & $S D$ & Meam & $S D$ \\
\hline Mid VC ratio & $1 \cdot 02^{*}$ & 0.22 & 0.80 & $0 \cdot 17$ \\
\hline Vmax 50 (I)/FVC (seconds) & $1 \cdot 28^{*}$ & 0.31 & $1 \cdot 59$ & 0.25 \\
\hline Vmax 50 (E)/FVC (seconds) & $1 \cdot 26$ & 0.24 & $1 \cdot 29$ & 0.32 \\
\hline Vmax 25 (E)/FVC (seconds) & 0.67 & $0 \cdot 15$ & 0.66 & 0.21 \\
\hline FVC' & $108 \cdot 40$ & $11 \cdot 58$ & $107 \cdot 87$ & 10.48 \\
\hline $\mathrm{FEV}_{1} \dagger$ & $102 \cdot 19$ & 9.79 & $105 \cdot 13$ & $9 \cdot 13$ \\
\hline $\mathrm{FEV}_{1} / \mathrm{FVC}^{\circ}$ & 89.5 & $5 \cdot 71$ & $87 \cdot 57$ & 6.09 \\
\hline PEFR/FVC (seconds) & $1 \cdot 86$ & $0 \cdot 30$ & $2 \cdot 00$ & 0.27 \\
\hline
\end{tabular}

*Indices which differed significantly between the two groups. †Percentage of predicted normal value.

the control group. The mean values in both groups were compared using Student's $t$ test. The mean mid VC ratios in the two groups (laryngomalacia $1.02 \pm 0.22$, control $0.80 \pm 0.17)$ differed significantly $(P<0 \cdot 001)$. Similarly the mean values for the $\dot{V} \max 50$ (I)/FVC ratios (laryngomalacia $1 \cdot 28 \pm$ $0 \cdot 31$, control $1.59 \pm 0.25$ second) were significantly different $(\mathrm{P}<0.001)$. Mean values for all other indices were comparable in both groups.

\section{Discussion}

The first case of laryngomalacia to be reported was in 1851 when Rilliet ${ }^{8}$ described a case of stridor in a young infant. In 1853 Rilliet and Barthez, ${ }^{9}$ in their textbook of diseases of children, included a description of a benign form of stridor in infants. Lees ${ }^{10}$ described a case of obstructed inspiration in 1883, and as the child subsequently died of diphtheria, he was able to describe the folded epiglottis and approximated arytenoepiglottic folds typical of the disorder. Sutherland and Lack ${ }^{11}$ described 18 cases 
of 'congenital laryngeal obstruction' in which they detailed the natural history and laryngoscopic appearances of this disorder. This description was further elaborated by Thomson and Logan Turner. ${ }^{12}$ Many names have been used for the disorder. The term 'laryngomalacia' was introduced by Jackson, ${ }^{13}$ (malacia being from the Greek malakia, meaning morbid softening of a part or tissue).

The natural history of laryngomalacia has been described. 111415 The wide variation in onset and in the duration of stridor is supported by our data. In this series the mean duration of stridor was 4 years 2 months and in 10 children it persisted beyond 2 years, the eldest child being 12 years 7 months. The previously noted association with respiratory infections $^{13}$ was confirmed. An association not previously described was persistent posseting (gastro-oesophageal reflux was strongly suspected in 6 children) resolving by 12 months.

The flow volume loop was found to be a useful method of assessing airway mechanics in laryngomalacia. There was a highly significant difference in the mean mid VC ratio between the laryngomalacia and control populations ( 1.02 and 0.80 respectively) and also in the mean Vmax 50 (I)/FVC ratios (1.28 and 1.59 seconds respectively), $\mathrm{P}<0.001$ for both indices. These indices reflect impairment of inspiratory flow alone and the mean values of all indices derived from the expiratory curve were normal in the study group.

Jordanoglou and Pride ${ }^{2}$ described a flow volume loop in an adult with tracheal stenosis. The loop showed a shallow inspiratory curve and a flattened expiratory curve with a mid VC ratio of $1 \cdot 16$. Miller and $\mathrm{Hyatt}^{3}$ studied obstructing lesions of the upper airways by measuring flow volume loops with patients breathing through orifices of different diameters representing different resistances. They demonstrated distinct patterns for fixed and variable intra- and extrathoracic obstruction, and derived normal values for mid VC ratios in adults (male $0.91 \pm 0.21$, female $0.99 \pm 0.23$ ). The pattern in fixed extrathoracic obstruction is the same as that described by Jordanoglou and Pride. ${ }^{2}$ Variable extrathoracic obstruction produces a shallow inspiratory curve and a normal expiratory curve resulting in a high mid $\mathrm{VC}$ ratio. The severity of obstruction varies with differences in transmural pressure. During inspiration the intramural pressure is subatmospheric, and the extramural pressure atmospheric; these produce narrowing of the airways. Intraluminal pressure is abnormally low on the alveolar side of the obstruction, producing turbulence of air flow, and an additional decrease in intraluminal pressure occurs from a Bernoulli effect caused by acceleration of air past the narrowing. Conversely, during expiration intraluminal pressure is positive compared with extraluminal pressure which tends to dilate the airways and alleviate the obstruction.

Thus 6 children who had laryngomalacia in infancy had flow volume loops consistent with variable extrathoracic inspiratory obstruction, and Case 1 had fixed extrathoracic airways obstruction, consistent with subglottic stenosis.

Because the pathophysiology in Case 1 was different, statistical analysis was repeated with his results excluded. The difference in mid VC and $\dot{V} \max 50$ (I)/FVC ratios was unchanged at $\mathrm{P}<0.001$. It was not possible accurately to predict those children with abnormal lung function tests from historical data, although they did tend to be boys with an early onset of stridor which persisted beyond $2 \frac{1}{2}$ years.

These findings demonstrate that laryngomalacia is not necessarily a benign self-limiting disorder. Stridor may persist well beyond 2 years. Although only Case 1, who had subglottic stenosis, had air flow limitation sufficient to alter his life style, it has been demonstrated that children with previous laryngomalacia may have persisting inspiratory airways obstruction which, if respiratory function is compromised, could place them at risk of respiratory decompensation necessitating earlier mechanical support of their airways than would otherwise be necessary. All children in the study group were prepubertal. It is possible that laryngeal growth at puberty may be sufficient to overcome the tendency to inspiratory laryngeal collapse.

\section{References}

1 Phelan P D, Gillam G L, Stocks J G, Williams H E. The clinical and physiological manifestations of the infantile larynx. Natural history and relationship to mental retardation. Aust Paediatr J 1971 ; 7 : 135-40.

2 Jordanoglou J, Pride N B. A comparison of maximal inspiratory and expiratory flow in health and in lung disease. Thorax 1968; 23: 38-45.

3 Miller R D, Hyatt R E. Obstructing lesions of the larynx and trachea. Clinical and physiologic characteristics. Mayo Clin Proc 1969; 44: 145-61.

4 Pride N B. The assessment of airflow obstruction. Role of measurements of airways resistance and of tests of forced expiration. Br J Dis Chest 1971 ; 65: 135-69.

5 Roncoroni A J, Goldman E, Puy R J M. Respiratory mechanics in upper airway obstruction. Bull Physiopathol Respir 1975; 11 : 803-22.

6 Rotman H H, Liss H P, Weg J G. Diagnosis of upper airway obstruction by pulmonary function testing. Chest 1975; 68: 796-9.

7 Polgar G, Promadhattavedi V. Pulmonary function testing in children: techniques and standards. Philadelphia: Saunders, 1971.

8 Rilliet F. De la dyspepsie acescente, consideree comme cause predisposante des affections choleriques. Revue Medico-chiurgicale 1851; July; 5-380. 
9 Rilliet F, Barthez E. Traite clinique et pratique des maladies des enfants, second edition. Vol. 1. Paris: Germer Eailliere, 1853: 485.

10 Lees D B. Larynx from an infant which had been the subject of a peculiar form of obstructed inspiration. Trans Pathol Soc London 1883; 34: 19-20.

11 Sutherland G A, Lack H L. Congenital laryngeal obstruction. Lancet 1897 ; ii: $653-5$.

12 Thomson J, Logan Turner A. On the causation of the congenital stridor of infants. Br Med J 1900; ii: 1561-3.

13 Jackson C, Jackson C L. Diseases and injuries of the larynx. New York: Macmillan, 1942.
14 Apley J. The infant with stridor. A follow up survey of 80 cases. Arch Dis Child 1953; 28: 423-35.

15 McSwiney P F, Cavanagh N P C, Languth P. Outcome in congenital stridor (laryngomalacia). Arch Dis Child 1977; 52: 215-8.

Correspondence to $\mathrm{Dr}$ G J Smith, Adelaide Children's Hospital, King William Road, North Adelaide, South Australia 5006.

Received 2 January 1980

The following articles will appear in future issues of this journal:

Cardiovascular risk factors in childhood C G D Brook

Cerebral structure and intraventricular haemorrhage in the neonate: a real-time ultrasound study M I Levene, J S Wigglesworth, and V Dubowitz

Factors causing rickets in institutionalised handicapped children on anticonvulsant therapy $Y$ Morijiri and T Sato

Relationship of exercise-induced asthma to clinical asthma in childhood $L$ Balfour-Lynn, M Tooley, and $S$ Godfrey

Breast development in the newborn J F McKiernan and D Hull

Immotile cilia syndrome: a new cause of neonatal respiratory distress $A$ Whitelaw, $A$ Evans, and $B$ Corrin

Use of the child health clinic $H$ Hart, $M$ Bax, and S Jenkins

Head insulation and heat loss in the newborn $J K$ Stothers 